\title{
Stabilizing a terahertz quantum-cascade laser using near-infrared optical excitation
}

\author{
Tasmim Alam ${ }^{1}$, Martin Wienold ${ }^{1,2}$, Xiang Lü ${ }^{3}$ Lutz Schrottke ${ }^{3}$, Holger T. Grahn ${ }^{3}$, Heinz-Wilhelm Hübers ${ }^{1,2}$ \\ ${ }^{1}$ German Aerospace Center (DLR), Institute of Optical Sensor Systems, Rutherfordstr. 2, 12489 Berlin, Germany \\ ${ }^{2}$ Humboldt-Universität zu Berlin, Department of Physics, Newtonstr. 15, 12489 Berlin, Germany \\ ${ }^{3}$ Paul-Drude-Institut für Festkörperelektronik, Leibniz-Institut im Forschungsverbund Berlin e. V., Hausvogteiplatz 5-7, \\ 10117 Berlin, Germany
}

\begin{abstract}
We demonstrate a technique to simultaneously stabilize the frequency and output power of a terahertz quantumcascade laser. The technique exploits frequency and power variations upon near-infrared excitation and does not require an external terahertz optical modulator. By locking the frequency to a molecular absorption line, we obtain a linewidth of about $260 \mathrm{kHz}$ and root-mean-square power fluctuations as low as $0.03 \%$.
\end{abstract}

\section{INTRODUCTION}

Terahertz (THz) quantum-cascade lasers (QCLs) are of great importance as local oscillators (LOs) for high-resolution heterodyne spectroscopy above $3 \mathrm{THz}$. Heterodyne receivers are commonly used for astronomical and atmospheric observations in the $\mathrm{THz}$ range [1]. The frequency resolution and radiometric accuracy of such a system depend on the frequency and output power stability of the LO. Increasing the stability of the LO with respect to frequency and output power has a direct impact on the performance of a heterodyne receiver. To resolve individual lines directly, a high-resolution heterodyne spectrometer requires a sub-MHz linewidth. Recent studies have shown that the quantum-noise limit for QCLs is in the sub-kHz range [2]. However, the practical linewidth for a freerunning THz QCL is much larger, which is mainly due to the limited stability of the injection current and operating temperature as well as vibrations in case of operation inside a mechanical cryocooler. In practice, the emission frequency drift can exceed more than $10 \mathrm{MHz}$ [3, 4]. In this work, we describe a method that allows for a simultaneous stabilization of the frequency and output power. The method takes advantage of near-infrared excitation for frequency and power regulation and does not require an external terahertz optical modulator.

\section{EXPERIMENTAL SETUP}

The experimental configuration is shown as a schematic representation in Fig. 1. The QCL at $3.1 \mathrm{THz}$ is operated inside a mechanical cryocooler, and the temperature is stabilized at $45 \mathrm{~K}$. The exit window of the cooler is made of high-density polyethylene (HDPE). A poly-4-methylpentene-1 (TPX) lens collimates the QCL beam, and a Mylar beam splitter divides the beam into two, where the reflected beam is used for power stabilization, while the transmitted beam passes through a gas cell containing methanol $\left(\mathrm{CH}_{3} \mathrm{OH}\right)$ vapor at a pressure of 1 hPA for frequency locking to a molecular absorption line. Via an optical fiber, the rear facet of the QCL is illuminated with a near-infrared (NIR) diode laser as for optical tuning $[5,6]$. The DC current for the QCL is provided by a laser driver (QCL1000 LAB). The driver has a typical modulation bandwidth of up to $3 \mathrm{MHz}$ and a modulation coefficient of 200
$\mathrm{mA} / \mathrm{V}$. For measuring the frequency stability with a lock-in amplifier, the QCL driving current is modulated by a sinusoidal signal at about $1 \mathrm{MHz}$ with a relatively small amplitude compared to the DC bias current. The signal from the lock-in amplifier corresponds to the first derivative of the absorption signal. An error signal is generated from the derivative of a spectroscopic signal which is proportional to the difference between the desired set point and the actual laser frequency. This error signal is used for the proportional-integral derivative (PID) controller to generate a modulation offset that is added to the QCL bias voltage to keep the error signal at zero and thus stabilizes the frequency to a particular methanol absorption line. A second PID controller, together with a diode laser, is used to stabilize the QCL output power.

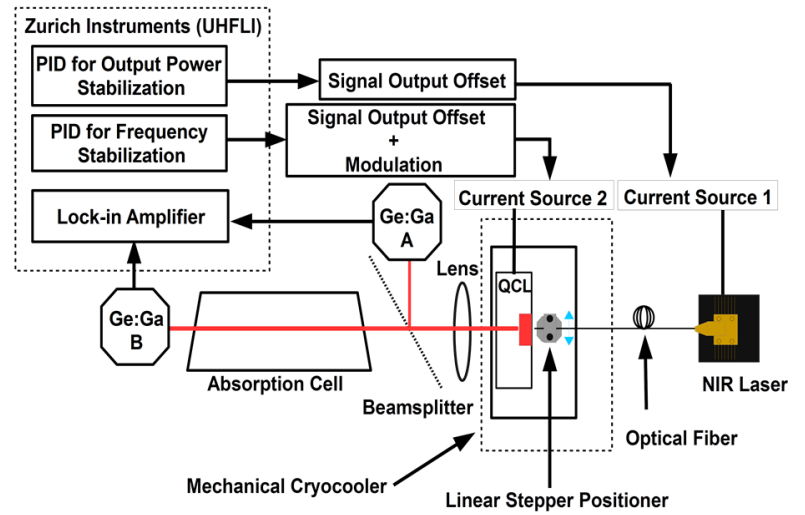

Fig. 1. Schematic setup for the frequency and output power stabilization.

All the signals are acquired with a fast data acquisition device (CDAQ, National Instruments), where both, input and output, channels are synchronized with a lock-in amplifier at $10 \mathrm{MHz}$ clock rate. Only proportional (P) and integral (I) parameters from the PID loop were used in this experiment. The proportional gain corrects the large quantities of the error in a small time interval, and the integral part sums the error over time and is used to correct a possible residual offset.

\section{RESULTS}

To characterize the stabilization, we monitor the frequency and the output power fluctuations of the QCL for three different modes. The frequency and output power stability for (1) a free-running QCL, (2) a frequency-locked QCL, and (3) both, a frequency- and power-locked, QCL are shown in Figs. 2(a) and 2(b), respectively. The frequency and power fluctuations in the free running mode are due to the variations in temperature and mechanical vibrations in the cryocooler. 
When the frequency stabilization loop is enabled, the frequency becomes very stable, while fluctuations in the QCL output power are even increased. In the third mode, when both PID control loops are simultaneously activated, the power and frequency remain stable.

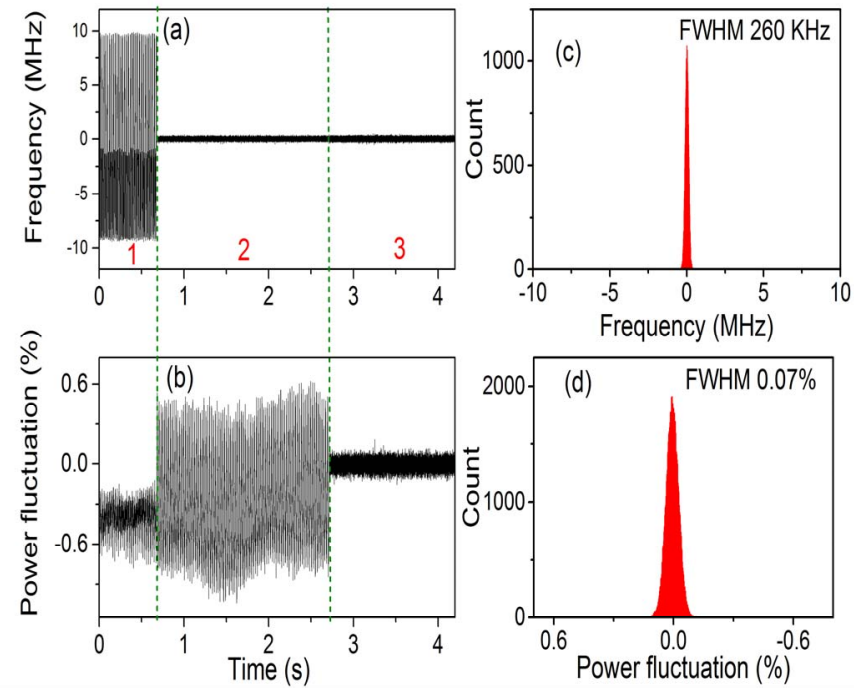

Fig. 2. (a) Frequency and (b) power fluctuations for three different operating modes: (1) a free-running QCL, (2) a frequency-locked QCL, and (3) a both, frequency- and power-locked, QCL. Distribution of the (c) frequency and (d) power fluctuations when both, the frequency and power, are stabilized.

To quantify the frequency stability, the voltage variations of the error signal need to be translated into frequency variations. Since the if signal has a linear dependence on the QCL driving current, the error signal can be converted directly to a frequency scale. The relative frequency and output power distribution for the frequency- and power-stabilized QCL are shown Figs. 2(c) and 2(d), respectively. We calculated a frequency variation of more than $10 \mathrm{MHz}$ in the free-running state. Technically, this is the laser frequency variation in the acquisition bandwidth (dc to $25 \mathrm{kHz}$ ) measured over a time interval of $0.7 \mathrm{~s}$. For the fully locked QCL, we found a Gaussian distribution with a full width at half maximum (FWHM) of approximately $260 \mathrm{kHz}$. We also measured the noise level of the $\mathrm{Ge}: \mathrm{Ga}$ detector $\mathrm{B}$ by blocking the terahertz radiation in front of the detector and record the noise-related lock-in signal. We found that the detector noise exhibits a Gaussian shape with a FWHM of $200 \mathrm{KHz}$ in a five-second time interval. This clearly indicates that the intrinsic noise level of the detector contributes significantly to the obtainable linewidth. To characterize the amplitude stabilization, the DC output of the Ge:Ga detector $\mathrm{A}$ is used as a power reference. As the frequency stability, the amplitude stability was recorded in the three experimental situations: In cases (1) and (2), the PID control loop for power stabilization was inactive, while it was active in case (3). For the free-running mode (1), the fluctuation corresponds to a root-mean-square (rms) stability of $0.1 \%$. For the situation (2), where only the frequency stabilization loop is active, this value even increases to $0.4 \%$. When the power control loop is switched on in case (3), the fluctuation of the QCL power is reduced to $0.03 \% \mathrm{rms}(0.07 \%$ FWHM) and exhibits a Gaussian distribution. This is more than a three-fold improvement with respect to the free-running case and more than a ten-fold improvement with respect to case (2), where just the frequency is stabilized.

\section{SUMMARY}

We successfully demonstrated the simultaneous stabilization of frequency and output power of a THz QCL operated in a compact cryocooler by means of a fiber-coupled optical excitation approach. The setup is compact and robust due to direct coupling of an optical fiber for illumination so that no external $\mathrm{THz}$ optical modulator is required to regulate the frequency and the output power. This scheme could play a vital role in improving the performance for high-resolution heterodyne spectroscopic systems.

\section{ACKNOWLEDMENTS}

T. Alam acknowledges the support by the Helmholtz Research School on Security Technologies.

\section{REFERENCES}

[1]. C. Risacher, R. Güsten, J. Stutzki, H. Hübers, D. Büchel, U. U. Graf, S. Heyminck, C. E. Honingh, K. Jacobs, B. Klein, T. Klein, C. Leinz, P. Pütz, N. Reyes, O. Ricken, H. Wunsch, P. Fusco, and S. Rosner, "First Supra-THz Heterodyne Array Receivers for Astronomy With the SOFIA Observatory," IEEE Trans.Terahertz Sci. Technol. vol. 6, pp.199-211, 2016.

[2]. M. S. Vitiello, L. Consolino, S. Bartalini, A. Taschin, A. Tredicucci, M. Inguscio, and P. De Natale, "Quantum-limited frequency fluctuations in a terahertz laser," Nat. Photonics vol. 6, pp. 525-528, 2012.

[3]. A. Barkan, F. K. Tittel, D. M. Mittleman, R. Dengler, P. H. Siegel, G. Scalari, L. Ajili, J. Faist, H. E. Beere, E. H. Linfield, A. G. Davies, and D. A. Ritchie, "Linewidth and tuning characteristics of terahertz quantum cascade lasers," Opt. Lett. vol. 29, pp. 575-577, 2004.

[4]. S. Barbieri, J. Alton, H. E. Beere, E. H. Linfield, D. A. Ritchie, S. Withington, G. Scalari, L. Ajili, and J. Faist, "Heterodyne mixing of two farinfrared quantum cascade lasers by use of a point-contact Schottky diode," Opt. Lett. vol. 29, pp. 1632-1634, 2004.

[5]. M. Hempel, B. Röben, L. Schrottke, H.-W. Hübers, and H. T. Grahn, "Fast continuous tuning of terahertz quantum-cascade lasers by rear-facet illumination," Appl. Phys. Lett. vol. 108, 2016, Art. no. 191106.

[6]. T. Alam, M. Wienold, X. Lü, K. Biermann, L. Schrottke, H. T. Grahn, and H.-W. Hübers, "Wideband, high-resolution terahertz spectroscopy by light-induced frequency tuning of quantum-cascade lasers," Opt. Express vol. 27 , pp. 5420-5432, 2019. 